\title{
A Case Report of Embryonal Botryoid Rhabdomyosarcoma of the Uterine Cervix in a 26- Year-Old Woman
}

\section{Gunel ZIYADOVA ${ }^{1}$, Altan KAVUNCUOGLU ${ }^{2}$, Gonca OZTEN¹, Zafer Selcuk TUNCER ${ }^{1}$}

Ankara, Turkey

\begin{abstract}
Botryoid rhabdomyosarcoma originating from the cervix is rare among fertile women and constitutes $0.2 \%$ of all uterine cancers. A 26 -year-old female patient with a cervical polyp presented with a case of sarcoma botryoides. The biopsy specimen confirmed the botryoid sarcoma of the uterine cervix and the patient underwent definitive surgery with lymphadenectomy. Pathology showed negative margins and total extraction of tumor. The tumor was confined to the cervix. The patient is considered group $1 \mathrm{~A}$ in Intergroup rhabdomyosarcoma Working Group V (IRSG) criteria and the treatment plan continued with adjuvant vincristine, actinomycin $\mathrm{D}$, cyclophosphamide chemotherapy.

In the case of cervical polyps in the fertile age, the specimen must be carefully and histologically examined to rule out genital rabdomyosarcoma. Since the stage is a very important prognostic factor, an early diagnosis is crucial.
\end{abstract}

Keywords: Sarcoma botryoides, Embryonal rhabdomyosarcoma, Vincristine, Actinomycin D, Cyclophosphamide protocol

Gynecol Obstet Reprod Med 2020;26(2)151-154

\section{Introduction}

Soft tissue sarcomas are a heterogeneous group of malignant diseases that show differentiation potential to striated or smooth muscle, nerve, fat, vascular tissues and other supporting tissues arising from primitive mesenchyme. Rhabdomyosarcoma (RMS) is the most common soft tissue sarcoma in children, constituting more than half of childhood soft tissue sarcomas. The average age of onset is 5-years-old; and the disease has two peaks between the ages of 2-6 and 15-

${ }^{1}$ Department of Obstetrics and Gynecology Hacettepe University, Ankara

${ }^{2}$ Department of Patology, Hacettepe University, Ankara

Address of Correspondence: Gunel Ziyadova

Department of Obstetrics and

Gynecology, Hacettepe University,

06230 Ankara, Turkey

drgunel87@gmail.com,

Submitted for Publication:

23. 03. 2018

Accepted for Publication:

19. 10.2018

\begin{tabular}{|c|c|}
\hline Quick Response Code: & Access this article online \\
\cline { 2 - 2 } & Website: www.gorm.com.tr \\
e- mail: info@gorm.com.tr
\end{tabular}

How to cite this article: Ziyadova G. Kavuncuoglu A. Ozten G. Tuncer ZS. A Case Report of Embryonal Botryoid Rhabdomyosarcoma of the Uterine Cervix in a 26-Year-Old Woman. Gynecol Obstet Reprod Med. 2020;26(2): 151-154
19. RMS is a very rare disease, particularly in the adult population. RMS can occur at different sites including: head, neck, genitourinary regions and extremities $(1,2)$.

The tumor is histologically divided into embryonal, alveolar, sclerosing and pleomorphic types. Embryonal is the most frequently observed histologic type, with three subtypes; botryoid, spindle cell, and not-otherwise-specified. The botryoid type means "grape-like" which is taken from the word "botrys" in Greek translations (3).

Botryoid tumor originating from the cervix among fertile women is rare and constitutes $0.2 \%$ of all uterine cancers. Oppositely to the childhood variant (mean age 23.5 months), which most frequently affects vagina, the uterus is the most common site for this tumor in post menopause and fertile age (mean age 14-18 years). Botryoid tumors of cervical origin have better prognosis than other regions of the female genital tract (4-6).

Current treatment of RMS is surgery with or without adjuvant chemotherapy or radiotherapy. The most appropriate treatment for patients with locally advanced disease is fertility sparing surgery combined with chemotherapy.

Here we report a case of a 26-year-old fertile woman, who developed sarcoma botryoides of the cervix uteri. 


\section{Case Report}

A 26-year-old female patient, without any medical illness, except postpartum venous thrombosis history, was admitted to the clinic with a complaint of vaginal mass sagging a month ago. During the pelvic examination of the patient, a $5 \mathrm{~cm}$ polyp on the cervix was observed macroscopically. The patient underwent polypectomy with local anesthesia. The pathological examination diagnosed the condition as RMS of the cervix. The tumor was $9 \mathrm{~cm}$ in size, the surgical margin was not detectable in some tumoral areas, with the botryoid type embryonal RMS in some areas and with alveolar RMS histology in other areas.

MRI and PET-CT scans were performed on the patient. There was no lesion in the cervical canal in MRI images (Figure 1). Heterodontic FDG uptake in the uterus and in the adnexal areas were observed in physiological limits. There was no evidence of any metastases.
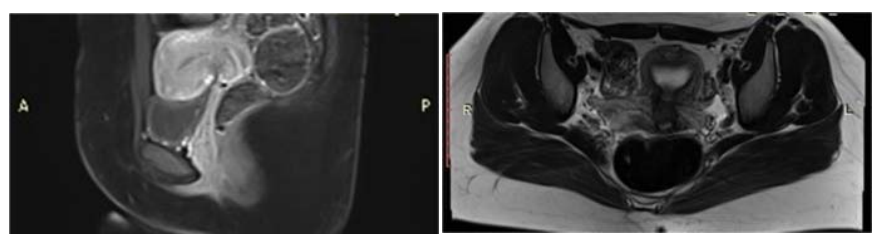

Figure 1: T2W post contrast fat saturated sagittal and T2W pre contrast non-fat-saturated axial MRI images clearly depicts intact stroma of cervix

Fertility sparing and definitive surgery with lymphadenectomy options were discussed with the patient. The patient was informed about the probability of the recurrence of illness and its prognosis. Since the patient has one child, the patient was offered fertility surgery option as well. Since she has no desire of fertility, definitive staging surgery was planned based off her decision.

Total abdominal hysterectomy, bilateral salpingo oophorectomy, pelvic lymph node sampling, washing cytology and omental biopsy were performed. During the operation, uterus, bilateral tubes and ovaries were all normal. No gross tumor and acid observed in the abdomen. The tissues removed during the surgery were sent to pathology. Final pathological diagnosis: RMS of the cervix, no macroscopic cervical mass was detected, and the cervical tumoral mass was observed in multiple microscopic foci on sections, the surgical margins were intact (Figure 2,3). In our patient's pathology material, tumor myogenin, myo-D1, vimentin, desmin with: broad positive, EMA, PLAP: focal positive: CD99, HMB45, S100, SMA, CK7, chromogranin: negative staining (Figure 4).

According to the IRSG criteria, the patient was evaluated to have the group IA disease (7). Adjuvant VAC (vincristine, dactinomycin and cyclophosphamide) chemotherapy was planned to reduce the risk of recurrence. The patient successfully completed the first round of chemotherapy. Informed consent was obtained from the patient.

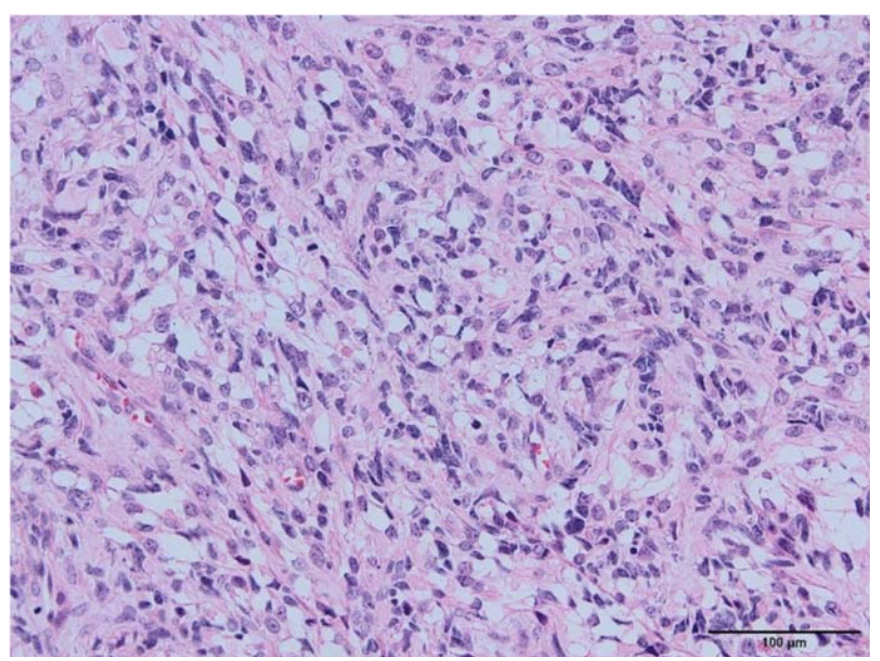

Figure 2: Neoplastic population consisted of undifferentiatedlooking cells, which had spindled oval nuclei with scant cytoplasm. 20x.

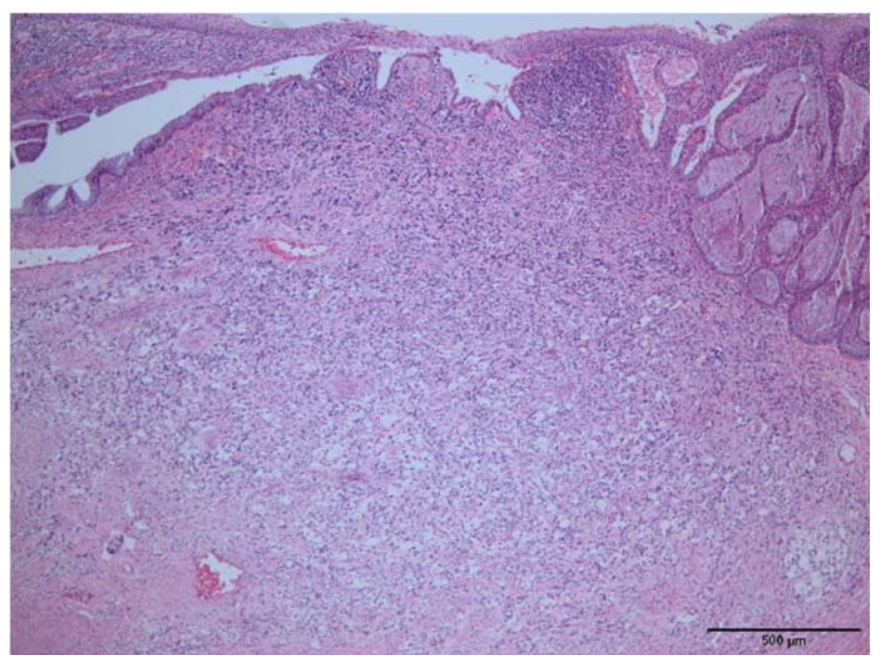

Figure 3: Microscopic findings revealed a hypercellular neoplasm that was located right beneath the endocervical epithelium and had irregular infiltrative borders in the stroma. Tumor hypercellularity was prominent superficially. $4 x$.

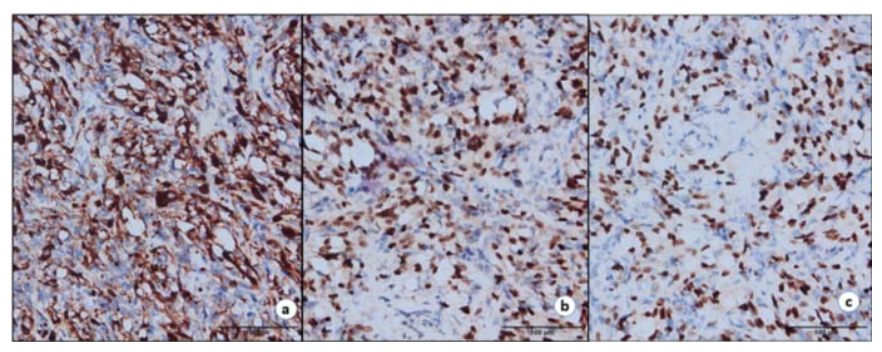

Figure 4: Immunohistochemical studies revealed that neoplastic cells were positive with Desmin (a), Myogenin (b) and Myo-D1 (c). Morphological and immunohistochemical results support the diagnosis of rhabdomyosarcoma. 20x.

\section{Discussion}

Approximately $90 \%$ of RMS cases occur in individuals under 25 years of age, and in this age group, $60-70 \%$ are under 10 years of age (8). Even if $20 \%$ of RMS occur in the geni- 
tourinary system in childhood, the cervix is a rare zone even in children and adolescence. The incidence of RMS in cervical cancers is between $1 \%$ and $0.4 \%$ (9).

Embryonal rhabdomyosarcoma of the cervix is a very rare disease that is seen mostly in case reports. The pathological study of cervical botryoid sarcoma in 13 women aged 12-26 years by Daya and Scully RE is noteworthy. These authors first stated that the sarcoma botryoides originated from uterine cervix in fertile women (10). As in many cases in the literature and in our case, the tumor is initially observed as a benign appearing cervical polyp. The patient's main complaints are the mass and hemorrhage bulging from the vagina (11). Immunohistochemical analysis (IHC) is essential for a confirmatory diagnosis. IHC markers, which label RMS, are desmin, muscle actinin and myoglobin with positivity rates of $75-100 \%, 78-97 \%$ and $28-67 \%$, respectively (12). In our case, tumor was myogenin, myo-D1, vimentin, desmin: diffuse positive, EMA, PLAP: focal positive: CD99, HMB45, S100, SMA, CK7, chromogranin: negative staining. Desmin, actin and myoglobin positivity are due to differentiation of the tumor with low sensitivity and specificity when poorly differentiated. On the other hand, Myo-D1 and myogenin are the most specific markers (70-100\% of cases) (13).

Prognosis depends on tumor size, depth of invasion and involvement of the lymph nodes. Tumors originating from the cervix have a better prognosis than other regions of the female genital tract. Embryonal botryoid variant has a much more favorable prognosis than alveolar and undifferentiated subtypes. Early stage is another positive prognostic factor, whereas metastatic disease and adjuvant chemotherapy requirement correlated with a poor prognosis (6).

The appropriate treatment of cervical sarcoma botryoides has not yet been well evaluated and should be individualized. At the beginning of 1970 s, definitive surgery involving pelvic lymphadenectomy was accepted as the first choice, but a significant increase in survival has not been proven. The IRSG has radically altered treatment protocols by adding surgical treatment adjuvant chemotherapy since 1972: the disease-free survival increased from $25 \%$ to $70 \%$ in 1985 . The 5 -year survival of patients in the IRS-III was approximately $93 \%$ when treated with vincristine and actinomycin (VA) for a year. Thus, the IRSG showed that chemotherapy increased survival (14).

Current treatment of RMS is surgery with or without adjuvant chemotherapy or radiotherapy. The appearance of the tumor in young women varies from simple local excision to definitive surgery with lymphadenectomy, since most of these women do not complete fertility. The most appropriate treatment for patients with locally advanced disease is fertility sparing surgery combined with chemotherapy. Four studies published by the IRSG in this regard, compared aggressive surgery (hysterectomy) and intensive radiotherapy with primary chemotherapy (VAC) and there was no significant change in survival was reported ( $82 \%$ for 5 -year survival / radiation and $84 \%$ for chemotherapy). If there is extensive uterine involvement, deep myometrial invasion, lymphatic involvement, presence of metastasis, or if the tumor is alveolar subtype, aggressive surgical treatment should be planned (15).

In our case, although our patient had one child, she had no fertility desire and decided to undergo aggressive surgery. In the cervical polyp pathology, the tumor showed botryoid type and alveolar RMS histology in some areas that need a further treatment.

Surgery and chemotherapy are essential in cervical RMS treatment and the prognosis of patients treated with multimodal therapy is very good (6).

Radiation therapy might be considered in patients with advanced age, recurrent disease and patients cannot tolerate chemotherapy (6).

In conclusion, the cervical polyp specimens belonging to fertile women must be carefully and histologically examined and should always be considered to rule out RMS. Diagnosis of RMS is extremely important, since early stage disease recognition is a very important prognostic factor. Surgery and chemotherapy are the mainstays of cervical RMS treatment and the prognosis of patients treated with multimodal therapy is expected to be good.

Acknowledgement: None.

Funding: No funding was used for this study

Conflict of Interest Statement: The authors state no conflict of interest

\section{References}

1. Wexler L, Helman L, Williams C. Principles and Practice of pediatric oncology. Edited by Pizzo PAPoplack DG. Lippincott-Williams \& Wilkins; 2002.

2. Kebudi R, Ağaoğlu F. Rabdomyosarkom. Klinik Gelişim Dergisi 2007;20(2):83-94.

3. Wright JD, Rosenblum K, Huettner PC, Mutch DG, Rader JS, Powell MA, et al. Cervical sarcomas: an analysis of incidence and outcome. Gynecologic Oncology 2005; 99(2):348-51.

4. Hilgers RD. Pelvic exenteration for vaginal embryonal rhabdomyosarcoma: a review. Obstetrics and gynecology $1975 ; 45(2): 175-80$

5. Maurer HM, Crist W, Lawrence W, Ragab AH, Raney RB, Webber B, et al. The intergroup rhabdomyosarcoma study I. A final report. Cancer 1988;61(2):209-20.

6. Walterhouse DO, Pappo AS, Meza JL, Breneman JC, Hayes-Jordan AA, Parham DM, et al. Shorter-duration therapy using vincristine, dactinomycin, and lower-dose cyclophosphamide with or without radiotherapy for patients with newly diagnosed low-risk rhabdomyosarcoma: 
a report from the Soft Tissue Sarcoma Committee of the Children's Oncology Group. Journal of Clinical Oncology 2014;32(31):3547.

7. Crist WM, Anderson JR, Meza JL, Fryer C, Raney RB, Ruymann FB, et al. Intergroup rhabdomyosarcoma studyIV: results for patients with nonmetastatic disease. Journal of Clinical Oncology 2001;19(12):3091-102.

8. Baiocchi G, Faloppa CC, de Toledo Osório CAB, Kumagai LY, Fukazawa EM, Cunha IW. Embryonal rhabdomyosarcoma of the uterine cervix in a 47 year old woman. Journal of Obstetrics and Gynaecology Research 2011;37(7):940-6.

9. Khosla D, Gupta R, Srinivasan R, Patel FD, Rajwanshi A. Sarcomas of uterine cervix: clinicopathological features, treatment, and outcome. International Journal of Gynecological Cancer 2012;22(6):1026-30.

10. Daya DA, Scully RE. Sarcoma botryoides of the uterine cervix in young women: a clinicopathological study of 13 cases. Gynecologic oncology 1988;29(3):290-304.

11. Bernal KL, Fahmy L, Remmenga S, Bridge J, Baker J. Embryonal rhabdomyosarcoma (sarcoma botryoides) of the cervix presenting as a cervical polyp treated with fertility-sparing surgery and adjuvant chemotherapy. Gynecologic oncology 2004;95(1):243-6.

12. Palazzo JP, Gibas Z, Dunton CJ, Talerman A. Cytogenetic study of botryoid rhabdomyosarcoma of the uterine cervix. Virchows Archiv A 1993;422(1):87-91.

13. Miyamoto T, Shiozawa T, Nakamura T, Konishi I. Sarcoma botryoides of the uterine cervix in a 46-year-old woman: case report and literature review. International journal of gynecological pathology 2004;23(1):78-82.

14. Raney RB, Maurer HM, Anderson JR, Andrassy RJ, Donaldson SS, Qualman SJ, et al. The Intergroup Rhabdomyosarcoma Study Group (IRSG): major lessons from the IRS-I through IRS-IV studies as background for the current IRS-V treatment protocols. Sarcoma 2001;5(1):9-15.

15. Sanders MA, Gordinier M, Talwalkar SS, Moore GD. Embryonal rhabdomyosarcoma of the uterine cervix in a 41-year-old woman treated with radical hysterectomy and adjuvant chemotherapy. Gynecologic oncology 2008;111 (3):561-3. 